\title{
Modeling of Survival and Frequency of Cardiovascular-Related Hospitalization in Patients with Transthyretin Amyloid Cardiomyopathy Treated with Tafamidis
}

\author{
Camille Vong ${ }^{1} \cdot$ Martin Boucher $^{2} \cdot$ Steve Riley $^{3} \cdot$ Lutz O. Harnisch $^{2}$ (I)
}

Accepted: 19 January 2021 / Published online: 26 March 2021

(c) The Author(s) 2021

\begin{abstract}
Introduction ATTR-ACT (Tafamidis in Transthyretin Cardiomyopathy Clinical Trial) demonstrated the efficacy and safety of tafamidis in transthyretin amyloid cardiomyopathy (ATTR-CM). Model-based analyses from ATTR-ACT can examine predictor effects on dose-response/exposure-response relationships.

Methods Parametric hazard distributions were developed for all-cause mortality and frequency of cardiovascular-related hospitalization. Time-to-event models were fitted to survival data, and repeated time-to-event models were fitted to hospitalization data. Disease-specific characteristics were assessed as baseline predictors of event hazards.

Results There were 441 patients in this analysis. At month 30, 70.5\% (tafamidis) and 57.1\% (placebo) of patients were alive, with 154/441 deaths reported; 495 cardiovascular-related hospitalizations occurred. The cumulative risk of death was $42.1 \%$ (95\% confidence interval [CI 24.2-58.0) lower with tafamidis than with placebo, regardless of New York Heart Association (NYHA) class; significant predictors of decreased risk were genotype (wild-type), greater 6-Minute Walk Test (6MWT) distance, higher left ventricular ejection fraction (LVEF), and lower blood urea nitrogen (BUN) and N-terminal pro-B-type natriuretic peptide concentrations. The average cumulative risk of cardiovascular-related hospitalization up to 30 months was $40.8 \%$ (95\% CI 31.0-49.7) lower with tafamidis in NYHA class I/II patients. Significant predictors of reduced risk were greater 6MWT distance, higher LVEF, and lower BUN and troponin I concentrations.

Conclusions Tafamidis reduced cumulative mortality and hospitalization risk versus placebo in patients with ATTR-CM. Baseline predictors of outcome were consistent with the cardiovascular nature of the disease and suggested that earlier treatment may improve outcomes.
\end{abstract}

Clinical Trials.gov Identifier NCT01994889 (date of registration: November 26, 2013).

\section{Key Points}

We conducted model-based analyses of predictors of death and cardiovascular-related hospitalization in ATTR-ACT (Tafamidis in Transthyretin Cardiomyopathy Clinical Trial).

Tafamidis reduced the risk of death (by 42.1\%), regard-

Digital Features To view digital features for this article go to https://doi.org/10.6084/m9.figshare.14219828.

Lutz O. Harnisch

Lutz.O.Harnisch@pfizer.com

1 Clinical Pharmacology, Pharmacometrics, Pfizer Inc, Cambridge, MA, USA

2 Clinical Pharmacology, Pharmacometrics, Pfizer Inc, Sandwich, UK

3 Clinical Pharmacology, Pfizer Inc, Groton, CT, USA less of New York Heart Association (NYHA) class, and the risk of cardiovascular-related hospitalization (by $40.8 \%$ ) in NYHA class I/II patients versus placebo.

Genotype (wild-type transthyretin amyloidosis [ATTRwt]) and greater 6-Minute Walk Test distance were predictors of reduced risk, as were higher left ventricular ejection fraction and lower blood urea nitrogen, $\mathrm{N}$-terminal pro-B-type natriuretic peptide, and troponin I concentrations. 


\section{Introduction}

Transthyretin amyloidosis (ATTR amyloidosis) is a rare, life-threatening disorder caused by the deposition of amyloid fibrils composed of misfolded transthyretin (TTR), which can accumulate in various organs and tissues within the body and primarily lead to progressive ATTR amyloidosis with polyneuropathy (ATTR-PN) or transthyretin amyloid cardiomyopathy (ATTR-CM) [1, 2]. Deposition of amyloid fibrils in the myocardium leads to ATTR-CM, characterized by a restrictive diastolic dysfunction and heart failure [3-5]. This deposition can be due to wild-type transthyretin (ATTRwt) or can occur in those with an inherited autosomal dominant mutation (variant) in the TTR gene (ATTRv) [5].

Tafamidis meglumine is an oral small molecule that binds to the thyroxine-binding sites on the TTR tetramer, stabilizing it and preventing dissociation into monomers and subsequent misfolding and aggregation as amyloid fibrils [6]. First shown to be an effective treatment for patients with ATTR-PN [7-10], tafamidis is approved in more than 40 countries for the treatment of adults with stage 1 symptomatic ATTR-PN [11]. The efficacy and safety of tafamidis in patients with ATTR-CM were demonstrated in ATTR-ACT (Tafamidis in Transthyretin Cardiomyopathy Clinical Trial), a phase III, multicenter, three-arm, parallel-design, placebocontrolled, randomized study [12, 13]. Tafamidis was shown to significantly reduce all-cause mortality and frequency of cardiovascular-related hospitalization. Tafamidis is approved for the treatment of both patients with ATTRv with ATTRCM and those with ATTRwt with ATTR-CM [14, 15].

The aim of these analyses of data from ATTR-ACT was to examine the risk for all-cause mortality and cardiovascular-related hospitalization in patients receiving tafamidis or placebo using a model-based approach and to evaluate the effects of predictive/prognostic factors on dose- or exposure-response relationships.

\section{Methods}

\subsection{Trial Design and Objectives}

The study design and primary results of ATTR-ACT (NCT01994889) have been published previously [12, 13]; in brief, patients were randomized 2:1:2 to receive tafamidis $80 \mathrm{mg}$, tafamidis $20 \mathrm{mg}$, or placebo for 30 months, respectively. The primary efficacy measures were all-cause mortality and the frequency of cardiovascular-related hospitalization, hierarchically assessed according to the Finkelstein-Schoenfeld method [16].

The current analyses sought to (1) examine the relationship between tafamidis $20 \mathrm{mg}$ or $80 \mathrm{mg}$ and placebo on all-cause mortality and frequency of cardiovascular-related hospitalization using a model-based approach and (2) evaluate the effects of predictive/prognostic factors (i.e., covariates) in the dose- and/or exposure-response relationship of all-cause mortality and cardiovascular-related hospitalization.

\subsection{Analyses}

All patients in ATTR-ACT who had at least one post-baseline efficacy evaluation (hospitalization, study visit, or date of death) were included in the analysis. The final datasets included subject identification, dosing information, time of efficacy events, longitudinal tafamidis pharmacokinetic exposure metrics taking into account dose reductions and dose interruptions, demographics, and patient disease characteristics.

The analyses used the same efficacy endpoints as the primary analysis [12]: all-cause mortality (with heart transplant or implantation of a cardiac mechanical assist device counted as death) and frequency of cardiovascular-related hospitalization over the 30-month duration of the trial. The frequency of cardiovascular-related hospitalization was defined as the number of times a subject was hospitalized for a cardiovascular-related morbidity.

Parametric hazard distributions were used in all analyses. Time-to-event (TTE) and repeated TTE (RTTE) models [17] were developed to describe the cumulative probability of death and cardiovascular-related hospitalization up to 30 months. The mathematical definitions of the TTE and RTTE hazard functions can be found in Fig. S1 in the electronic supplementary material (ESM).

\subsubsection{Predictive/Prognostic Factor Analysis}

To assess clinically or statistically significant predictors for risk of death and risk of hospitalization, potential covariates, determined by prior literature search or clinical judgment, were graphically and descriptively explored with and without stratification. TTE and RTTE endpoints were plotted as Kaplan-Meier (KM) plots, along with median survival times, and confidence intervals (CIs) were stratified by categorical covariates of interest. KM mean covariate (KMMC) plots were used to evaluate continuous covariates, where the mean of each covariate for all individuals remaining in the study after every event occurring was plotted at every change point of a KM curve [18].

Covariates deemed influential by KMMC plots were evaluated for inclusion in the model and are described in Table 1. Covariate selection was guided using a stepwise covariate model-building procedure. After the covariate search, the impact of each retained covariate on the hazard was calculated. This was compared with the final selected composite set of baseline covariates, based on the coefficients estimated in the model. 


\subsubsection{Evaluation of Model Performance}

The predictive performance of parametric models used in TTE/RTTE analyses was evaluated using simulation-based KM visual predictive check (VPC) plots of the observed TTE data related to the $90 \%$ prediction interval calculated from simulations of 200 trials. For RTTE, a separate KM estimator was generated for time to first event, time to second event, etc. For both models, the VPCs were stratified by covariates of interest, including those not present in the model, to judge the overall predictive performance of the developed models.

Survival probability was also estimated by splitting each covariate (with the exception of genotype) into tertiles and displaying the resulting distribution of the survival probability within those bounds.

To introduce treatment effect and assess its magnitude given individual covariates, survival $[S(t)]$ was calculated for all patients under tafamidis treatment and under placebo treatment, and a hypothetical risk reduction (RR) was calculated for all patients in the trial using Eq. (1):

$\% \mathrm{RR}=100 \cdot\left(1-\frac{1-S(t)_{\text {tafamidis }}}{1-S(t)_{\text {placebo }}}\right)$

in which $S(t)$ was computed as the mean survival at month 30 under placebo or treatment (pooled tafamidis) and RR was derived representing the resulting hypothetical RR.

\section{Results}

\subsection{Observed Data: Modeling Dataset}

Patient demographics have been reported elsewhere [12]; in brief, most patients were male $(\sim 90 \%)$, with a median age of 75 years, and were predominantly ATTRwt $(\sim 76 \%)$ (Table $\mathrm{S} 1$ in the ESM). Patients had a mean \pm standard deviation (SD) 6-Minute Walk Test (6MWT) distance of $351.6 \pm 123.1 \mathrm{~m}$ and a mean left ventricular ejection fraction (LVEF) of $47.8 \pm 11.8 \%$. The mean concentration of blood urea nitrogen (BUN) was $29.2 \pm 13.2 \mathrm{mg} / \mathrm{dL}$, and the mean concentration of $\mathrm{N}$-terminal pro-B-type natriuretic peptide (NT-proBNP) was $461.1 \pm 379.6 \mathrm{pmol} / \mathrm{L}$.

The percentage of patients alive at month 30 was $70.5 \%$ with tafamidis and $57.1 \%$ with placebo. A total of 154 deaths were reported (including 13 patients who underwent a heart transplant or implantation of a cardiac mechanical assist device; these events were counted as death) (Table 2). The KM plot for all-cause mortality has been reported previously [12].

In total, there were 495 cardiovascular-related hospitalizations across all patients (range $0-8$ visits/patient; Table 2 ). Overall, average cardiovascular-related hospitalizations were 1.12 over 30 months/patient: 1.31 in the placebo group, 0.94 in the tafamidis $20-\mathrm{mg}$ group, and 1.03 in the tafamidis 80-mg group. A total of 96 (21.8\%) patients withdrew from the trial: $50(28.3 \%[n=50 / 177])$ from the placebo group, $14(15.9 \%)$ from the tafamidis $20-\mathrm{mg}$ group, and $32(18.2 \%)$ from the tafamidis $80-\mathrm{mg}$ group (Table 2).

\subsection{Modeling Results}

\subsubsection{Survival Hazard (Time to Event)}

A Gompertz time-varying hazard model best described the death hazard distribution in the placebo cohort. When analyzing the data including all tafamidis-treated patients, the pooled $20-\mathrm{mg}$ and $80-\mathrm{mg}$ effect demonstrated a reduction over time, indicating that tafamidis reduced the risk of death. VPCs demonstrated a good fit to the placebo, tafamidis 20-mg, and tafamidis $80-\mathrm{mg}$ data (Fig. 1; Fig. S2 in the ESM).

Estimating both dose effects separately resulted in a trend toward a greater effect with $80 \mathrm{mg}$, though no differentiation between doses could be estimated.

Table 1 Description of potential covariates of the exposure-response analysis

\begin{tabular}{ll}
\hline Predictors & Description \\
\hline Demographics & $\begin{array}{c}\text { Age, height, weight, modified body mass index, sex, race, ethnicity, country or region (USA and ex-USA), alcohol con- } \\
\text { sumption, smoking status }\end{array}$ \\
Disease specific & $\begin{array}{r}\text { TTR genotype (variant and wild-type); NYHA class (classes I and II combined and class III); KCCQ-OS; EQ-5D-3L index } \\
\text { score, EQ-visual analog score; summary metrics related to the duration of cardiovascular-related hospitalization; cumula- } \\
\text { tive cardiovascular-related hospitalization count; 6MWT }\end{array}$ \\
& $\begin{array}{l}\text { Respiration rate } \\
\text { Vital signs }\end{array}$ \\
Laboratory values & $\begin{array}{l}\text { NT-proBNP, troponin I, BUN, albumin } \\
\text { Echocardiogram }\end{array}$ \\
& $\begin{array}{l}\text { End-diastolic interventricular septal wall thickness, left ventricle posterior wall thickness, left ventricular ejection fraction, } \\
\text { left ventricular stroke volume, global longitudinal strain, circumferential strain mid global, radial strain mid global }\end{array}$ \\
\hline
\end{tabular}

$6 M W T$ 6-Minute Walk Test, $B U N$ blood urea nitrogen, $E Q-5 D-3 L$ EuroQoL five-dimensions, three-level questionnaire, $K C C Q-O S$ Kansas City Cardiomyopathy Questionnaire Overall Score, NT-proBNP N-terminal pro-B-type natriuretic peptide, NYHA New York Heart Association, TTR transthyretin 
Table 2 Summary of death, dropout, and cardiovascular-related hospitalization visits

\begin{tabular}{|c|c|c|c|c|c|}
\hline Event & $\begin{array}{l}\text { Placebo } \\
(n=177)\end{array}$ & $\begin{array}{l}\text { Tafamidis } 20 \mathrm{mg} \\
(n=88)\end{array}$ & $\begin{array}{l}\text { Tafamidis } 80 \mathrm{mg} \\
(n=176)\end{array}$ & $\begin{array}{l}\text { Pooled tafamidis } \\
(n=264)\end{array}$ & $\begin{array}{l}\text { All patients } \\
(n=441)\end{array}$ \\
\hline All-cause mortality & $76(42.9)$ & $24(27.3)$ & $54(30.7)$ & $78(29.5)$ & $154(34.9)$ \\
\hline Dropout $^{\mathrm{a}}$ & $50(28.3)$ & $14(15.9)$ & $32(18.2)$ & $46(17.4)$ & $96(21.8)$ \\
\hline \multicolumn{6}{|c|}{ CV-related hospitalizations } \\
\hline 0 & $70(39.5)$ & $46(52.3)$ & $80(45.5)$ & $126(47.7)$ & $196(44.4)$ \\
\hline 1 & $53(29.9)$ & $22(25.0)$ & $46(26.1)$ & $68(25.8)$ & $121(27.4)$ \\
\hline 2 & $23(13.0)$ & $10(11.4)$ & $28(15.9)$ & $38(14.4)$ & $61(13.8)$ \\
\hline 3 & $16(9.0)$ & $5(5.7)$ & $15(8.5)$ & $20(7.6)$ & $36(8.2)$ \\
\hline 4 & $4(2.3)$ & $2(2.3)$ & $4(2.3)$ & $6(2.3)$ & $10(2.3)$ \\
\hline 5 & $3(1.7)$ & $2(2.3)$ & $1(0.6)$ & $3(1.1)$ & $6(1.4)$ \\
\hline 6 & $4(2.3)$ & 0 & $1(0.6)$ & $1(0.4)$ & $5(1.1)$ \\
\hline 7 & $3(1.7)$ & 0 & $1(0.6)$ & $1(0.4)$ & $4(0.9)$ \\
\hline 8 & $1(0.6)$ & $1(1.1)$ & 0 & $1(0.4)$ & $2(0.5)$ \\
\hline
\end{tabular}

Data are presented as $n(\%)$

$C V$ cardiovascular

${ }^{\text {a }}$ Dropout is only related to the recording of hospitalizations; there were no dropouts in the recording or follow-up on death

In the final TTE model, the estimated treatment effect was a cumulative hazard ratio of 0.579 , indicating a $42.1 \%(95 \%$ CI 24.2-58.0) reduction in the cumulative risk of death over 30 months with pooled tafamidis compared with placebo. Covariates (Table 1) were assessed to obtain the final model using KMMC plots. An example of the KMMC plots for both the base and the final TTE model assessing the influence of baseline 6MWT on the hazard are shown in Fig. S3 in the ESM; the KM plot for all-cause mortality has been reported previously [12].

Significant baseline predictors of survival were wildtype genotype, greater 6MWT distance, higher LVEF, and lower concentrations of BUN and NT-proBNP (Fig. 2). Final parameter values in the TTE model are shown in Table S2 in the ESM. These individual baseline covariates were able to improve the prediction of survival in the model, but their joint inclusion achieved the best characterization of the hazard in predicting survival. Based on the observed data, each covariate was split into tertiles (with the exception of genotype) to illustrate its impact on the distribution of the survival probability and the projected relative risk, demonstrating that patients with less severe disease had a greater risk reduction with tafamidis, and again indicating that the composite provided an improved prediction compared with individual factors (Fig. 3; Fig. S4 in the ESM).

The inclusion of baseline New York Heart Association (NYHA) class strata I/II or III did not improve the predictive model for TTE over the set of baseline covariates discussed. The tafamidis treatment effect was significant $(p<0.05)$ in both strata, though there was no significant difference in the treatment effect between the strata: $48.0 \%(95 \% \mathrm{CI}$
27.1-64.6) and 30.4\% (95\% CI 4.84-52.8) for NYHA class I/II and class III, respectively.

\subsubsection{Hospitalization Hazard (Repeated Time to Event)}

All hospitalization events per patient were modeled as a longitudinal individual risk assessment using an RTTE approach. The best fit to the underlying hospitalization hazard distribution was a Gompertz distribution, obtained by modeling the placebo data only, indicating that the risk of hospitalization increased over time.

The treatment effect was a $40.8 \%$ (95\% CI 31.0-49.7) reduction in the weighted average cumulative risk of cardiovascular-related hospitalization over 30 months in NYHA class I/II patients. A distribution function providing the probabilities of each subsequent event occurring before time $t$ stratified by treatment (Fig. 4) indicated that most of the treatment effect was observed between events 1 and 2, likely because of the larger numbers of these events compared with later events. While the RTTE model characterized the hospitalization data adequately, some model misspecification remained, as the second and third events were predicted to happen later than the data indicated (Fig. S5 in the ESM).

Differentiation of cardiovascular-related hospitalization hazard ratios based on tafamidis $20-\mathrm{mg}$ or $80-\mathrm{mg}$ doses or exposure were not estimable within the 30 -month trial duration.

Final parameter values in the RTTE model are shown in Table S2 in the ESM. Significant baseline predictors of decreased hospitalization hazard were greater 6MWT distance, higher LVEF, and lower concentrations of BUN and troponin I (Fig. 2). 
a
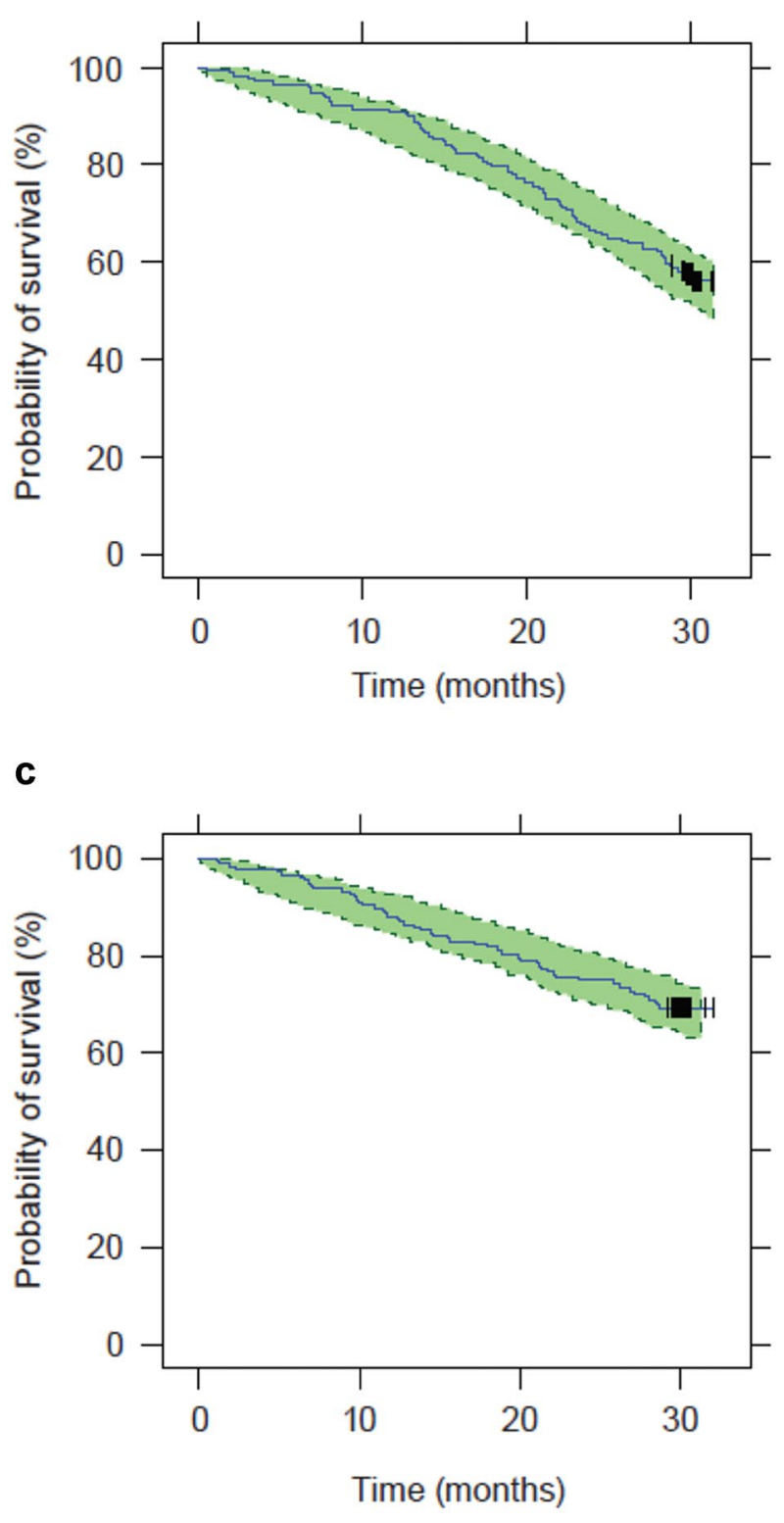

Fig. 1 Kaplan-Meier visual predictive check for the base time-toevent Gompertz survival model in a placebo, b tafamidis $20-\mathrm{mg}$, and c tafamidis 80-mg cohorts, comparing the observed data (line) to the

The inclusion of baseline NYHA class strata I/II or III did not improve the predictive model for RTTE over the set of baseline covariates discussed. But, in contrast to the treatment effect estimated in either NYHA class strata for TTE, a significant treatment effect was found only in NYHA class I/II $(p<0.01)$, whereas no treatment effect was found for NYHA class III.

\section{b}

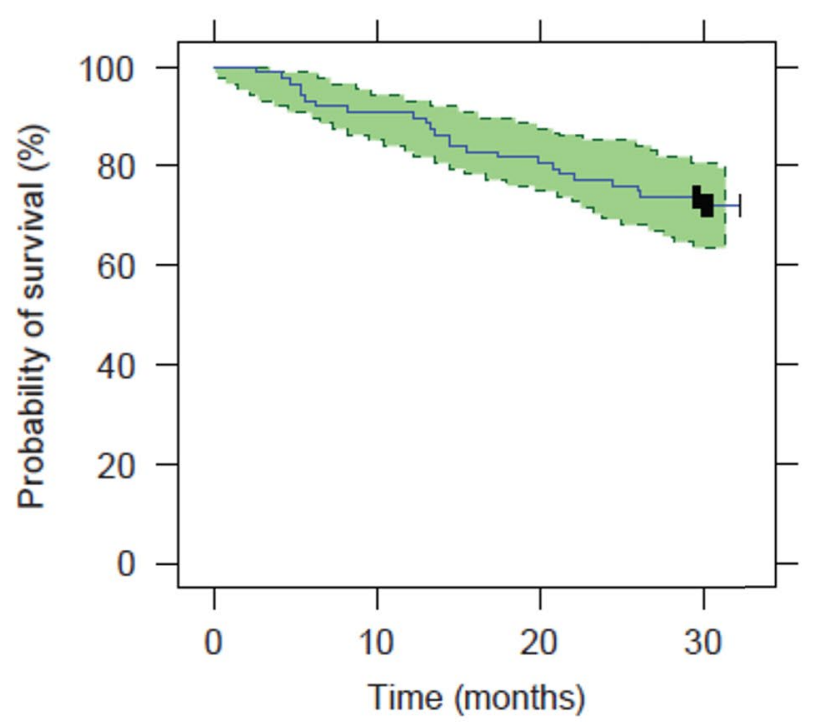

90\% prediction interval of the simulated data (green area) with the time-to-event model

\section{Discussion}

Risk of mortality and cardiovascular-related hospitalization in patients with ATTR-CM receiving tafamidis or placebo were both characterized by a time-varying hazard function following a Gompertz model, suggesting that the risk of an event occurring increases over time, which is a departure from the usual constant hazard assumption of a Cox proportional model. Results indicated that treatment with tafamidis was associated with a $42.1 \%$ reduction in cumulative risk of 


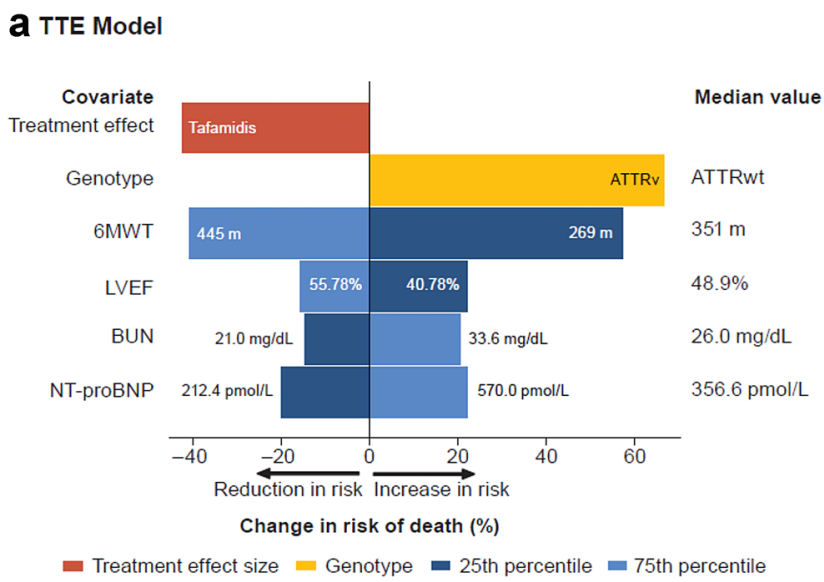

Fig. 2 Final model effects of identified covariates and the baseline hazards for a TTE (survival) and b RTTE (cardiovascular-related hospitalization). Figure shows the percentage change in risk of death with values for each covariate in the 25 th percentile or 75 th percentile, compared with the risk at their median values. Baseline incidence/ranges for covariates (total population): genotype, $24.0 \%$ ATTRv; 6MWT, 24-822 m; BUN, 8.7-129.0 mg/dL; LVEF, 11.0-

death regardless of NYHA class and a $40.8 \%$ reduction of cardiovascular-related hospitalization in NYHA class I/II patients up to 30 months.

Predictors of survival associated with lower risk of death were wild-type genotype, 6MWT distance, LVEF, and BUN and NT-proBNP concentrations; this indicates that a patient with ATTRwt, a greater 6WMT distance, higher LVEF, and lower BUN and NT-proBNP concentrations would have an increased likelihood of survival. While each of these factors was found to be predictive individually, the combination of these covariates improved prediction of the hazard versus any of the components individually.

Predictors associated with a lower risk of cardiovascularrelated hospitalization were 6MWT distance, LVEF, and BUN and troponin I concentrations. The predictive factors associated with cardiovascular-related hospitalization were similar to those associated with increased survival, indicating that a patient with a greater 6WMT distance, higher LVEF, and lower BUN and troponin I concentrations would likely have a decreased risk of a hospitalization over 30 months.

The predictors identified are in line with the well-recognized ATTR-CM patient profile described in the literature $[19,20]$. Previously, some analyses have failed to identify genotype as a risk factor for mortality [21]. However, reports have indicated left ventricular systolic dysfunction (ejection fraction $<50 \%$ ) as an independent predictor of survival in ATTR-CM [19], and serum troponin and NT-proBNP are recognized biomarkers for the assessment of myocardial involvement in amyloidosis [22, 23].

\section{b RTTE Model}

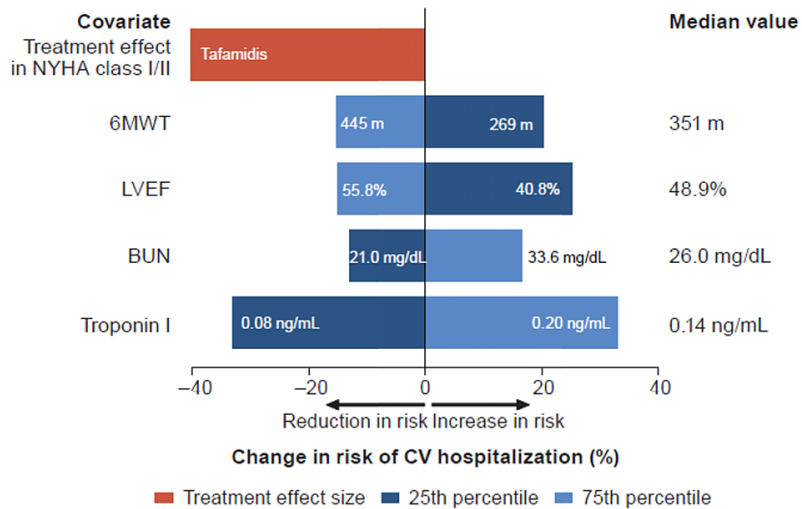

85.4\%; NT-proBNP, 35.2-2598.0 pmol/L; troponin I, 0.03-12.22 ng/ mL. 6MWT 6-Minute Walk Test, ATTRv variant transthyretin amyloidosis, ATTRwt wild-type transthyretin amyloidosis, $B U N$ blood urea nitrogen, $L V E F$ left ventricular ejection fraction, $N T$-proBNP N-terminal pro-B-type natriuretic peptide, NYHA New York Heart Association, RTTE repeated time-to-event, TTE time-to-event

Both the survival and the cardiovascular-related hospitalization models demonstrated that, consistent with the primary outcome in ATTR-ACT [12], patients with ATTR-CM treated with tafamidis had a clinically significant reduction in cumulative risk of mortality and of cardiovascular-related hospitalization up to month 30 . Within the 30 -month trial duration, no differentiation between tafamidis meglumine 20-mg and 80-mg doses or exposure was identified on either endpoint using these models, which allowed the test for the predictive value of the covariates and a dose differentiation assessment on each separate endpoint, in contrast to the primary analysis, which was not conducive to a dose-response assessment. ATTR-ACT was not designed to assess the relative efficacy of each tafamidis dose. Rather, the study was designed to compare pooled tafamidis with placebo, with at least $90 \%$ power to detect a $30 \%$ reduction in mortality, a reduction in the frequency of cardiovascular-related hospitalizations from 2.5 to 1.5 (over the 30 -month duration of the trial), or both [12].

However, greater TTR stabilization, reduction in the decline in NT-proBNP, and improved survival with longer exposure to tafamidis $80 \mathrm{mg}$ compared with tafamidis 20 $\mathrm{mg}$, supported the approval of $80 \mathrm{mg}$ as the recommended dose [14, 24].

It is well understood that more advanced disease, reflected by a higher baseline NYHA class, is associated with a higher chance of dying or being hospitalized. This idea was confirmed in the model by the fact that the final composite of baseline covariates also largely correlated with baseline NYHA class. As tafamidis treatment improved the outcome (reduced the risk of mortality) across the full range of the 
Fig. 3 Cumulative survival probability at month 30 given baseline characteristics from the final time-to-event model. The blue line represents the overall cumulative survival probability assuming all patients received placebo. Tertile distributions are represented for each covariate, with their respective range. $6 M W T$ 6-Minute Walk Test, $B U N$ blood urea nitrogen, COVBASE composite set of baseline covariates, $L V E F$ left ventricular ejection fraction, NT-proBNP N-terminal pro-Btype natriuretic peptide

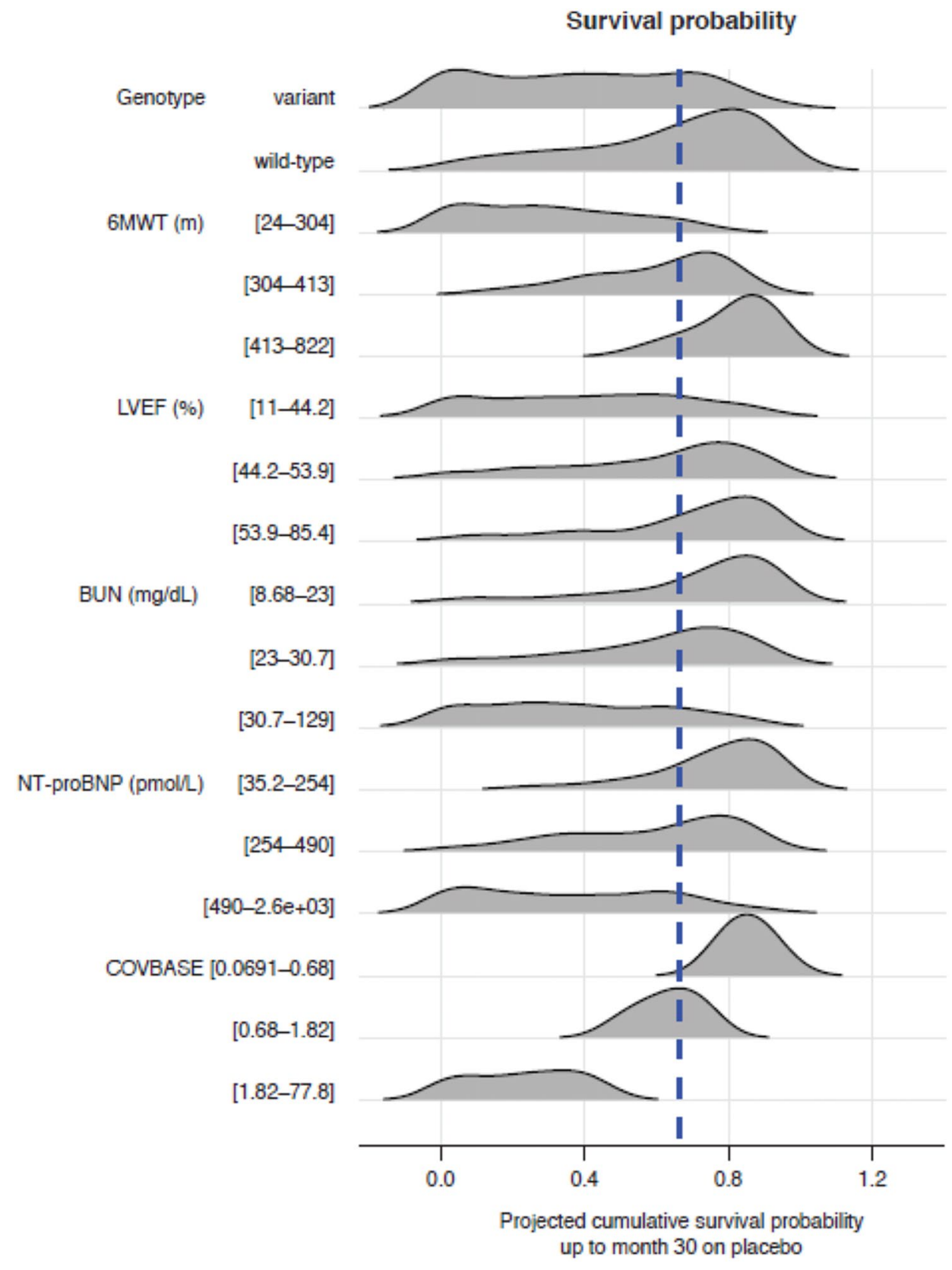


Fig. 4 Cumulative risk reduction over 30 months for each hospitalization event to occur with tafamidis. The dashed horizontal intercept is the overall reduction in the weighted average cumulative risk in New York Heart Association class I/II patients over 30 months treated with tafamidis. An individual hypothetical risk reduction was calculated based on 1000 simulated datasets using the model, treatment effect, and the design matrix of the trial with all patients on placebo then subsequently on tafamidis treatment. Since every simulation gives a different number of hospitalizations per patient, each trial simulation was summarized according to the frequency of events, and a weighted mean in the reduction was calculated based on the distribution of cumulative events. Each point on the graph corresponds to the risk reduction for the specific number of events, whereas the horizontal line takes the weighed mean approach

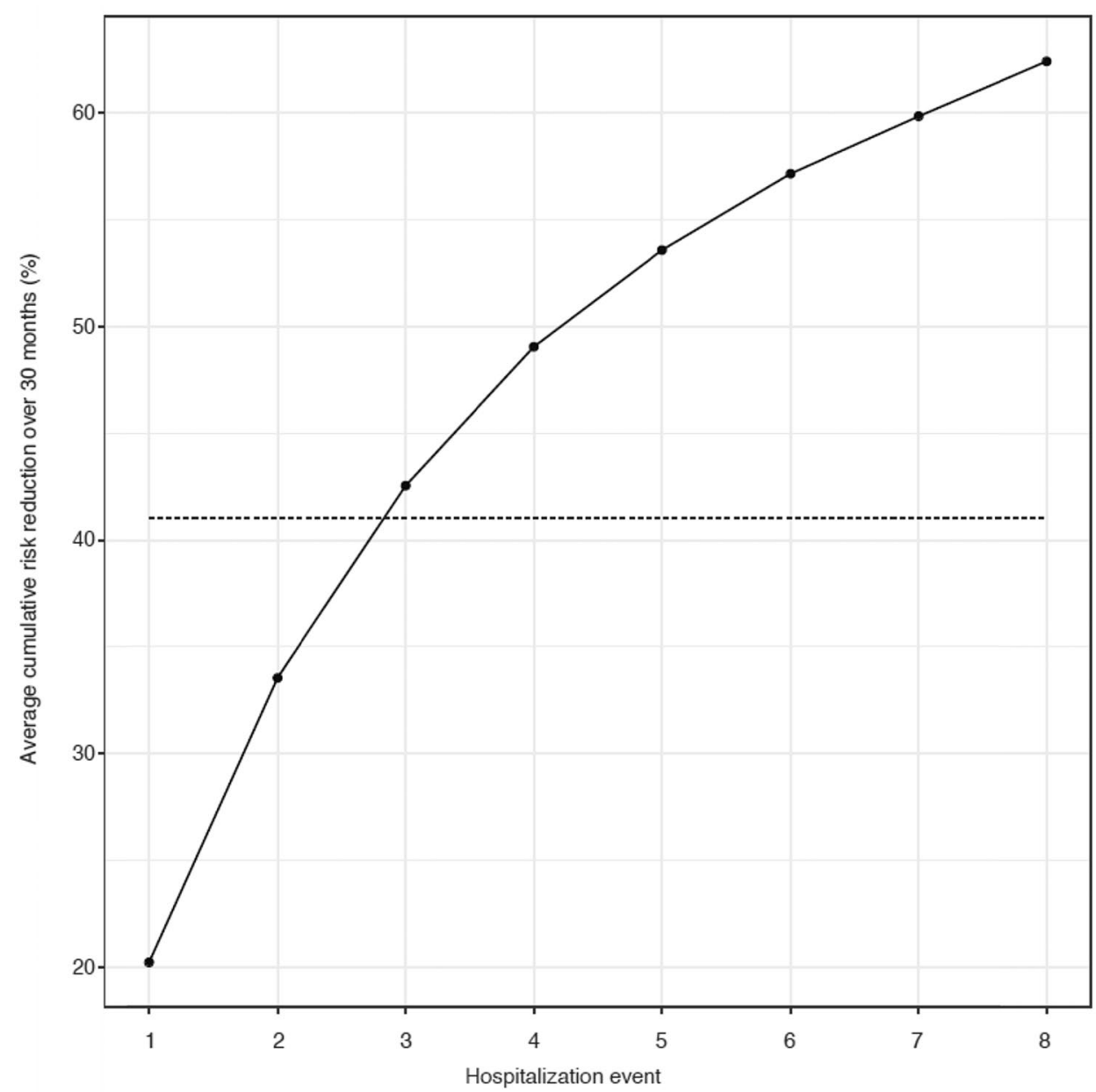

The RTTE data were censored by both death and dropout events, a situation that defines a semi-competing risk framework. Rather than competing, the rate of dropout may be occurring at random, increasing the range of variability. However, if the rate of hospitalization and TTE are correlated, death would be considered as competing, as those with fewer hospitalizations survive longer, whereas the observation period for those who die earlier is shorter, potentially leading to a biased estimator. Future analyses could address this issue using a joint modeling of the three events, in which likelihood of a hospitalization event would be defined as conditional on the probability of having survived and not dropped out up to the time of a hospitalization event, though this approach also involves an assumption regarding the nature of the correlation between these endpoints.

Assessment of cardiovascular echocardiogram is a useful tool for the assessment of ATTR-CM [25], and several echocardiogram variables were collected in ATTR-ACT [12]. Among all the baseline echocardiogram covariates examined in the model, global longitudinal strain baseline and LVEF were the two most significant predictors of mortality. Given the medical familiarity and interpretability of LVEF, this was the only predictor kept in the final TTE model, and increased LVEF was shown to be a significant baseline predictor of increased survival and of decreased cardiovascular-related hospitalization hazard.

These data support the primary outcome of ATTR-ACT, which demonstrated improved survival and reduced hospitalization risk for patients treated with tafamidis. The covariates identified as significant baseline predictors of outcome in the models suggest that treating patients with ATTR-CM earlier in the disease course may result in better outcomes.

Supplementary Information The online version contains supplementary material available at https://doi.org/10.1007/s40256-021-00464-y.

Acknowledgements Medical writing support was provided by Caitlin Watson, PhD, of Engage Scientific Solutions, and funded by Pfizer.

\section{Declarations}

Funding This analysis was sponsored by Pfizer.

Conflict of interest S. Riley, M. Boucher, and L.O. Harnisch are fulltime employees of, and hold stock and/or stock options with, Pfizer. C. 
Vong was a full-time employee of Pfizer at the time of the analysis; she is now a full-time employee of Novartis AG and holds stock options with Novartis AG.

Ethics approval ATTR-ACT was approved by the independent review board or ethics committee at each participating site and was conducted in accordance with the provisions of the Declaration of Helsinki and the International Council for Harmonisation Good Clinical Practice guidelines.

Consent to participate All patients provided written informed consent.

Consent for publication All authors have given their consent for the publication of this manuscript.

Availability of data and material Upon request, and subject to review, Pfizer will provide the data that support the findings of this study. Subject to certain criteria, conditions, and exceptions, Pfizer may also provide access to the related individual anonymized participant data. See https://www.pfizer.com/science/clinical-trials/trial-data-and-resul ts for more information.

\section{Code availability Not applicable.}

Author contributions All authors contributed to the design of the analysis, data acquisition and interpretation, and to the drafting and revising of the manuscript.

Open Access This article is licensed under a Creative Commons Attribution-NonCommercial 4.0 International License, which permits any non-commercial use, sharing, adaptation, distribution and reproduction in any medium or format, as long as you give appropriate credit to the original author(s) and the source, provide a link to the Creative Commons licence, and indicate if changes were made. The images or other third party material in this article are included in the article's Creative Commons licence, unless indicated otherwise in a credit line to the material. If material is not included in the article's Creative Commons licence and your intended use is not permitted by statutory regulation or exceeds the permitted use, you will need to obtain permission directly from the copyright holder. To view a copy of this licence, visit http://creativecommons.org/licenses/by-nc/4.0/.

\section{References}

1. Planté-Bordeneuve V, Said G. Familial amyloid polyneuropathy. Lancet Neurol. 2011;10:1086-97.

2. Gertz MA. Hereditary ATTR amyloidosis: burden of illness and diagnostic challenges. Am J Manag Care. 2017;23:S107-12.

3. Ruberg FL, Berk JL. Transthyretin (TTR) cardiac amyloidosis. Circulation. 2012;126:1286-300.

4. Connors LH, Sam F, Skinner M, Salinaro F, Sun F, Ruberg FL, et al. Heart failure resulting from age-related cardiac amyloid disease associated with wild-type transthyretin: a prospective, observational cohort study. Circulation. 2016;133:282-90.

5. Ruberg FL, Grogan M, Hanna M, Kelly JW, Maurer MS. Transthyretin amyloid cardiomyopathy: JACC state-of-the-art review. J Am Coll Cardiol. 2019;73:2872-91.

6. Bulawa CE, Connelly S, Devit M, Wang L, Weigel C, Fleming JA, et al. Tafamidis, a potent and selective transthyretin kinetic stabilizer that inhibits the amyloid cascade. Proc Natl Acad Sci USA. 2012;109:9629-34.

7. Coelho T, Maia LF, Martins da Silva A, Waddington Cruz M, Planté-Bordeneuve V, Lozeron P, et al. Tafamidis for transthyretin familial amyloid polyneuropathy: a randomized, controlled trial. Neurology. 2012;79:785-92.

8. Coelho T, Maia LF, da Silva AM, Cruz MW, Planté-Bordeneuve $\mathrm{V}$, Suhr OB, et al. Long-term effects of tafamidis for the treatment of transthyretin familial amyloid polyneuropathy. J Neurol. 2013;260:2802-14.

9. Barroso FA, Judge DP, Ebede B, Li H, Stewart M, Amass L, et al. Long-term safety and efficacy of tafamidis for the treatment of hereditary transthyretin amyloid polyneuropathy: results up to 6 years. Amyloid. 2017;24:194-204.

10. Coelho T, Merlini G, Bulawa CE, Fleming JA, Judge DP, Kelly JW, et al. Mechanism of action and clinical application of tafamidis in hereditary transthyretin amyloidosis. Neurol Ther. 2016;5:1-25.

11. Pfizer Limited. Tafamidis $20 \mathrm{mg}$ Summary of Product Characteristics. 2016. https://www.medicines.org.uk/emc/product/2837/ smpc. Accessed 12 June 2020.

12. Maurer MS, Schwartz JH, Gundapaneni B, Elliott PM, Merlini $\mathrm{G}$, Waddington-Cruz $\mathrm{M}$, et al. Tafamidis treatment for patients with transthyretin amyloid cardiomyopathy. N Engl J Med. 2018;379:1007-16.

13. Maurer MS, Elliott P, Merlini G, Shah SJ, Cruz MW, Flynn A, et al. Design and rationale of the phase 3 ATTR-ACT clinical trial (Tafamidis in Transthyretin Cardiomyopathy Clinical Trial). Circ Heart Fail. 2017;10:e003815.

14. Pfizer Inc. VYNDAQEL ${ }^{\circledR}$ (tafamidis meglumine) prescribing information. US Food and Drug Administration. 2019. https:// www.fda.gov/media/126283/download. Accessed 20 Sept 2019.

15. Pfizer Inc. Vyndaqel (tafamidis) $20 \mathrm{mg}$ and $61 \mathrm{mg}$ summary of product characteristics. European Medicines Agency. 2020. https:// www.ema.europa.eu/en/documents/product-information/vyndaqelepar-product-information_en.pdf. Accessed 12 June 2020.

16. Finkelstein DM, Schoenfeld DA. Combining mortality and longitudinal measures in clinical trials. Stat Med. 1999;18:1341-54.

17. Cox EH, Veyrat-Follet C, Beal SL, Fuseau E, Kenkare S, Sheiner LB. A population pharmacokinetic-pharmacodynamic analysis of repeated measures time-to-event pharmacodynamic responses: the antiemetic effect of ondansetron. J Pharmacokinet Biopharm. 1999;27:625-44.

18. Hooker AC, Karlsson MO. The Kaplan-Meier mean covariate plot (KMCC): a new diagnostic for covariates in time-to-event models. In: Annual Meeting of the Population Approach Group in Europe (PAGE); 2012; Venice, Italy.

19. Lee MH, Lee SP, Kim YJ, Sohn DW. Incidence, diagnosis and prognosis of cardiac amyloidosis. Korean Circ J. 2013;43:752-60.

20. Witteles RM, Bokhari S, Damy T, Elliott PM, Falk RH, Fine NM, et al. Screening for transthyretin amyloid cardiomyopathy in everyday practice. J Am Coll Cardiol Heart Fail. 2019;7:709-16.

21. Maurer MS, Hanna M, Grogan M, Dispenzieri A, Witteles R, Drachman B, et al. Genotype and phenotype of transthyretin cardiac amyloidosis: THAOS (Transthyretin Amyloid Outcome Survey). J Am Coll Cardiol. 2016;68:161-72.

22. Kyriakou P, Mouselimis D, Tsarouchas A, Rigopoulos A, Bakogiannis C, Noutsias M, et al. Diagnosis of cardiac amyloidosis: a systematic review on the role of imaging and biomarkers. BMC Cardiovasc Disord. 2018;18:221.

23. Kristen AV, Maurer MS, Rapezzi C, Mundayat R, Suhr OB, Damy $\mathrm{T}$. Impact of genotype and phenotype on cardiac biomarkers in patients with transthyretin amyloidosis-Report from the Transthyretin Amyloidosis Outcome Survey (THAOS). PLoS ONE. 2017;12:e0173086.

24. Damy T, Garcia-Pavia P, Hanna M, Judge DP, Merlini G, Gundapaneni B, et al. Efficacy and safety of tafamidis doses in the Tafamidis in Transthyretin Cardiomyopathy Clinical Trial (ATTRACT) and long-term extension study. Eur J Heart Fail. 2020.

25. Donnelly JP, Hanna M. Cardiac amyloidosis: an update on diagnosis and treatment. Cleve Clin J Med. 2017;84:12-26. 